\title{
Enhanced PAtient Clinical Streamlining (EPACS): Quality Initiative to Improve Healthcare for New Surgical Outpatient Visits
}

\author{
Elvira L. Vos, MD ${ }^{1}$, Jessica S. Cho, BA ${ }^{1}$, Joseph Schmeltz, BA ${ }^{2}$, Nick Teri, MS ${ }^{1}$, Ethel B. Law, MSN ${ }^{1}$, \\ Kathleen Paisley, MPAS ${ }^{1}$, Aaron Begue, MSN $^{3}$, Helen Loumeau, $\mathrm{MSN}^{4}$, Sherri H. Suozzo, MSN, PhD $^{5}$, \\ Latasha Anderson-Dunkley, MSN ${ }^{6}$, Ginger J. Gardner, MD ${ }^{6}$, Elizabeth Jewell, MD, MHSc ${ }^{6}$,
} Samuel Singer, $\mathrm{MD}^{1}$, Nadeem Abu-Rustum, $\mathrm{MD}^{6}$, William R. Jarnagin, $\mathrm{MD}^{4}$, Julio Garcia Aguilar, $\mathrm{MD} \mathrm{PhD}^{7}$, Jeffrey Drebin, $\mathrm{MD}^{4}$, and Vivian E. Strong, $\mathrm{MD}^{1}$

${ }^{1}$ Gastric and Mixed Tumor Service, Department of Surgery, Memorial Sloan Kettering Cancer Center, New York, NY;

${ }^{2}$ Technology Division, Memorial Sloan Kettering Cancer Center, New York, NY; ${ }^{3}$ Advanced Practice Providers Administration, Memorial Sloan Kettering Cancer Center, New York, NY; ${ }^{4}$ Hepatopancreatobiliary Service, Department of Surgery, Memorial Sloan Kettering Cancer Center, New York, NY; ${ }^{5}$ Thoracic Service, Department of Surgery,

Memorial Sloan Kettering Cancer Center, New York, NY; ${ }^{6}$ Gynecology Service, Department of Surgery, Memorial Sloan Kettering Cancer Center, New York, NY; ${ }^{7}$ Colorectal Service, Department of Surgery, Memorial Sloan Kettering Cancer Center, New York, NY

\begin{abstract}
Purpose. For patients who select a specialty hospital for cancer treatment, the wait time until the initial consultation leaves patients anxious and delays treatment. To improve quality of care, we implemented an enhanced patient clinical streamlining (EPACS) process that establishes an early connection and coordinates care before the first surgical outpatient visit at our specialty cancer center.

Methods. During a pre-visit EPACS phone call to new patients, an advanced practice provider (APP) collected medical history and ordered work-up tests or consultations if feasible. First visit cancellation rate, number of patients who started treatment, time to start of treatment, and satisfaction by the care team and patient were compared between patients treated with versus without EPACS.
\end{abstract}

Results. Among 5062 consecutive new patients, 720 (14\%) received an EPACS call and 4342 did not (86\%); work-up was ordered pre-visit in $34 \%$ and $16 \%$,

(C) Society of Surgical Oncology 2021

First Received: 17 July 2021

Accepted: 14 October 2021;

Published Online: 5 January 2022

V. E. Strong, MD

e-mail: strongv@mskcc.org respectively. Fewer EPACS patients cancelled the first visit ( $4.6 \%$ vs. $12 \%, p<0.001)$, more started treatment $(55 \%$ vs. $50 \%, p=0.037$ ), and their time to treatment was shorter, but not significantly (median 17 vs. 19 days, $p=0.086$ ). Patient interaction was considered to be improved by EPACS by 17 of 17 APPs and 14 of 16 surgeons, and outpatient clinic efficiency by 14 of 17 APPs and 13 of 16 surgeons. EPACS reduced anxiety and increased preparedness for the first visit in 29 of 31 patients.

Conclusions. EPACS improved effectiveness, timeliness, and physician and patient satisfaction with health care at our cancer center.

When patients receive a cancer diagnosis, they have many questions and face many life-altering decisions, leading to tremendous anxiety. Immediate decisions include where to get the best care and how quickly treatment can be started. While data support superior outcomes of cancer care provided by specialty centers, over threequarters of cancer patients are treated in community hospitals. ${ }^{1}$ A survey suggested that over $90 \%$ of the US public could be motivated to travel farther away for complex cancer surgery if informed of a safety or quality advantage. ${ }^{2}$ However, the majority of patients are highly anxious 
after their cancer diagnosis, ${ }^{3}$ perhaps affecting individuals' decisions for their care. A study from our center found that the key patient considerations when selecting a hospital for cancer care included recommendations by their doctor, familiarity with the hospital, preference for a specific doctor, minimal travel time, and starting treatment quickly (unpublished data).

Shorter time to treatment initiation has repeatedly been associated with better oncological outcomes, ${ }^{4}$ and reflects the resources available and efficiency of the overall care system at a hospital. ${ }^{5}$ An important barrier to rapid treatment is the wait time for an initial consultation. The time between diagnosis and first visit is generally not utilized efficiently, as often it is not until the day of outpatient clinic that familiarity with the provider is fostered, anxiety addressed, medical records reviewed, and care organized. Importantly, inefficiency of clinical work processes is a contributing factor to physician burnout. ${ }^{6}$ Therefore, establishing an early connection with the patient would improve both patients' and providers' experience, in addition to likely improving treatment outcomes.

In a previous pilot study, we reorganized existing resources to develop an early connection process for new patients at four surgical services at our cancer center. After a patient scheduled an initial consultation, an advanced practice provider (APP; nurse practitioner or physician assistant) performed pre-visit evaluations by phone to achieve familiarity, provide simple education, answer questions, and obtain medical history. Before the first visit, outside medical records, radiological imaging, and other evaluations were obtained and work-up ordered if feasible. This intervention shortened the median time to treatment from 20 days (interquartile range [IQR] 9-32) among the 72 patients who did not receive a pre-visit call to 15 days (IQR 9-27) for 100 patients who were called, and the proportion of patients with high anxiety before the first visit from 35 to $23 \%$ (data not published). Based on these encouraging results, positive feedback from APPs and surgeons, and unsolicited positive feedback from patients, we decided to expand and implement this early connection process as a standard of care.

In this quality improvement study, we implemented an improved version of this clinical streamlining process for new outpatient clinic patients, called Enhanced Patient Clinical Streamlining (EPACS), in four surgical services. In the present study, we evaluated the association between EPACS and effectiveness of care, timeliness of care, and health care satisfaction from providers' and patients' perspectives.

\section{METHODS}

\section{Study Design, Participants, and Setting}

This quality improvement study took place at the Department of Surgery at Memorial Sloan Kettering Cancer Center (MSK) in New York City between February 2020 and April 2021, and followed the Standards for QUality Improvement Reporting Excellence (SQUIRE) 2.0 guidelines on reporting interventions for improving quality of healthcare. ${ }^{7}$ Following institutional policies, Institutional Review Board approval was not required because this study was considered quality improvement. As this study could preferentially benefit those selected for EPACS by allowing treatment to start sooner, outcomes were evaluated after 1 year and were presented to departmental management to consider continuity and expansion of EPACS for all patients. Patients were eligible if they were newly seen at MSK for a primary cancer diagnosis or a high suspicion of cancer, were scheduled for an initial consultation with a surgeon on the Colorectal, Gastric and Mixed Tumor, Gynecology, or Hepatopancreatobiliary Service, and had been assigned a medical record number. Internal referrals and patients with metastatic disease were excluded. After careful introduction and education of EPACS to all parties involved, EPACS was launched on 6 February 2020. Because EPACS required a new workflow, APPs were instructed to start making several EPACS calls per week in a consecutive fashion from the list of eligible patients, with no minimum or maximum requirement. Surgical fellows who rotate among surgical services were also allowed to use EPACS when preparing for their outpatient clinic. Because surgical fellows contributed only a small proportion of patients, throughout the manuscript we refer only to APPs.

\section{Standard of Care/Non-enhanced Patient Clinical Streamlining (EPACS) Evaluation and Treatment Scheduling}

The standard of care for new patients at the participating surgical services was as follows (Fig. 1). After Patient Access Service schedules an appointment for new patients and collects the minimum required documentation, before alerting the surgeon's office coordinator (OC). Some OCs collect further documentation before the clinic visit, including radiological imaging and other evaluations such as pathological tissue slides. On the day of the first outpatient visit, the APP reviews available records and introduces themself and the care team, obtains medical history, answers questions, etc., after which the patient meets the surgeon. The APP and surgeon write orders for work-up, defined as radiology, consultations, blood tests, 
FIG. 1. EPACS workflow. EPACS Enhanced Patient Clinical Streamlining, $A P P$ advanced practice provider

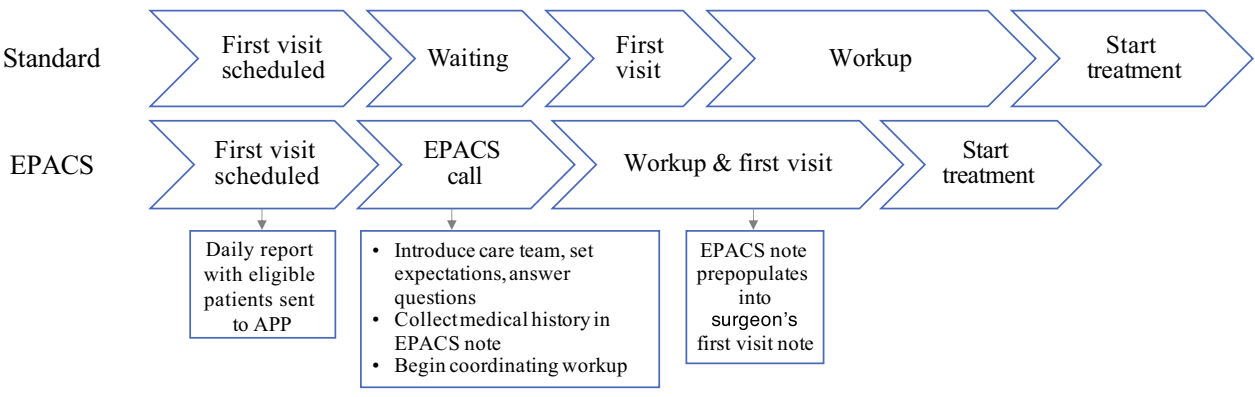

cardiology, endoscopy, and non-therapeutic interventional radiology, that are scheduled by the outpatient clinic nurse. Work-up is scheduled as soon as possible and on the same day as other work-up to the extent possible. For most patients, the goal is to start treatment as soon as possible.

\section{EPACS Supporting Resources}

EPACS required the development of several new resources. First, APPs were granted access to a shared folder with the OCs and Patient Access Service, where all patient documentation is saved until the electronic medical record (EMR) is available, which requires insurance clearance and hospital-specific medical record number assignment. Second, daily computer-automated reports were created and sent to APPs by email, listing all new patients scheduled for a first visit in their service. Third, an EPACS-specific note was created in the EMR to document the EPACS phone call, the duration of the call, and medical history collected on the call that automatically forwards and pre-populates into the APP's and surgeon's first visit note. Progress of EPACS implementation was assessed throughout the study period in regular meetings among the EPACS project management team, APP and OC managers, and other parties involved (e.g. Patient Access Service) to solicit feedback, present updates, and discuss adjustments to the EPACS protocol.

\section{EPACS Phone Call}

The APP selected a patient for the EPACS call from the daily report and reviewed the patient's records in the shared folder (Fig. 1). The EPACS phone calls were conducted at the APPs' discretion, but the call was supported by a script laid out as a checklist at the top of the EPACS note that included introducing themselves, the surgical service, and the care team; explaining the reason for the call; obtaining medical history; setting expectations for the first visit and likely course of action; reminding patients to send or bring relevant documents and evaluations; and answering questions. If feasible, and in consultation with the surgeon, the APP could begin coordinating care by ordering work-up to be scheduled by the OC before the first visit, on the same day, or after.

\section{Outcomes and Measures}

Process measures studied included the timing of the EPACS call, defined as the number of days prior to the first visit, and the duration of the EPACS call in minutes. The timing of orders for work-up was measured, defined as before the initial visit versus after. The primary outcome measures were the cancellation rate, time to treatment, and conversion rate. Cancellation rate was defined as the number of patients who cancelled a first visit that was not rescheduled within 30 days within the same service. The time to start treatment was counted in days from the first visit. Treatment was defined as surgery with curative intent, oral or intravenous chemotherapy, radiotherapy, or therapeutic interventional radiology. The conversion rate was defined as the number of patients who completed their first visit and started treatment within 3 months. Secondary outcome measures were differences in the administrative workload; quality of patient interaction; outpatient clinic efficiency in EPACS versus non-EPACS patients from the perspective of the OCs, APPs, and surgeons; and reduction in patient-reported anxiety and improvement in preparedness for the first visit.

\section{Data Collection and Statistical Analysis}

Patient demographic, clinical, and treatment characteristics, process measures, and primary outcome measures were collected through an extensive query of the institutional database. The database pulls data from the EPIC scheduling software Cadence and Optime and from the Allscripts clinical information application housing EMRs, among other sources. The institutional database was queried on 10 April 2021 for all eligible patients with a new visit between 10 February 2020, the day of the first visit of the first EPACS patient, and 9 April 2021. Patients who received an EPACS call and had an EPACS note in their EMR were defined as the EPACS group, and all other patients were defined as the non-EPACS group. Data 
accuracy was assessed by manually checking all variables for a random subset of 51 patients.

Secondary outcome measures were collected by surveys 3 months after launch. An electronic survey was emailed to all OCs who had organized care for EPACS patients, APPs who had called EPACS patients, and surgeons who had seen EPACS patients, evaluating the effectiveness of EPACS through the following questions: (1) "Do you feel a difference in administrative workload (patient phone calls, rescheduling work-up, etc.)?"; (2) "Do you feel a difference in patient interaction (perceived stress/anxiety, types of questions asked, etc.)?"; and (3) "Do you feel a difference in clinic efficiency?". Responses were graded on a 5-point scale: much improved, little improved, no difference, little worse, or much worse. The patient survey was administered by phone to randomly selected EPACS patients until 30 responded, asking: (1) "To what extent did this call help reduce your anxiety?"; and (2) "To what extent did this call help you feel organized and/or prepared for the initial consultation with the doctor?" on a scale of 1 to 10 (1 meaning not organized/prepared at all, and 10 meaning very organized/prepared for the visit).

Demographics, clinical and treatment characteristics, and process and outcome measures were compared between the EPACS and non-EPACS groups. Continuous variables were compared using the Mann-Whitney U test and categorical variables were compared using the Chisquare test. The effect of time was studied by comparing the percentage of EPACS patients among 3-month periods. Variation among the four surgical services was studied for primary but not secondary outcomes because of the limited number of participants. Secondary outcome measures were presented using descriptive statistics. Statistical analyses were performed using SPSS for Windows, version 27.0 (IBM Corporation, Armonk, NY, USA).

\section{RESULTS}

\section{Patient Characteristics}

Figure 2 shows the number of eligible and excluded patients. From the total of 5062 patients included, 720 (14\%) received an EPACS call and 4342 did not $(86 \%)$. The percentage of patients who received an EPACS call was consistent over time and ranged from 11 to $20 \%$ for each 3-month period (electronic supplementary Fig. 1). Patients in the non-EPACS and EPACS groups were similar in age, sex, and new visit type (telehealth or in-person) [Table 1]. While the largest number of patients were seen by the Gynecology Service (30\% of all patients included), the proportion of EPACS patients was greatest within the Gastric and Mixed Tumor Service (38\%). The distribution

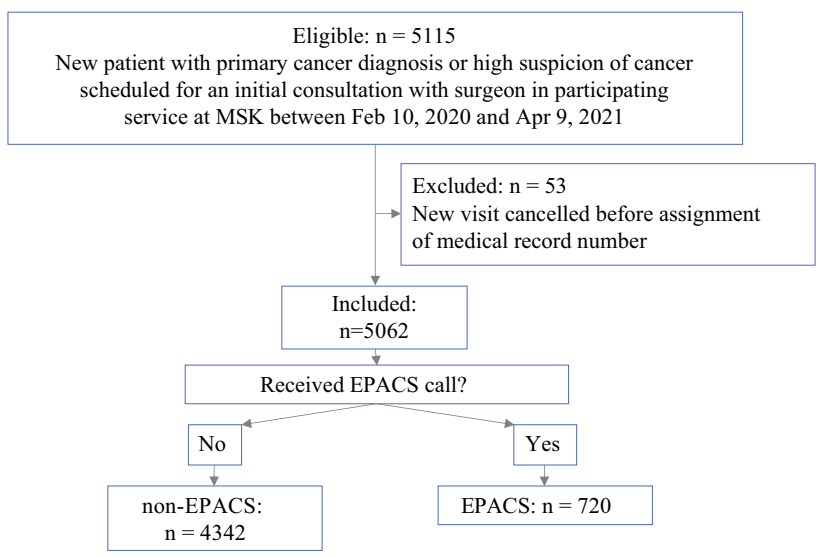

FIG. 2. Patient flow chart. EPACS Enhanced Patient Clinical Streamlining, MSK Memorial Sloan Kettering Cancer Center

of tumor sites at diagnosis was comparable between the non-EPACS and EPACS groups, except for pancreas and liver being more common in the non-EPACS group and stomach and rectal cancer being more common in the EPACS group. The data accuracy rate was $97.7 \%$.

\section{Process Measures}

The EPACS phone call was made at a median of 2 days (IQR 1-4) before the first visit by one of 18 APPs or 13 surgical fellows. Call duration was reported in the EPACS note in 655 of $720(91 \%)$ calls, and took $<5 \mathrm{~min}$ in $0.9 \%$ of calls, 5-10 min in 17\% of calls, 10-20 min in $48 \%$ of calls, 20-30 min in $24 \%$ of calls, and $>30 \mathrm{~min}$ in $10 \%$ of calls. The median time it took APPs to prepare for the phone call, perform the call, and organize care after the call, as reported by the APPs in the survey, was $38 \mathrm{~min}$. Of the 4525 patients who completed their first visit, 3906 (86\%) had work-up ordered before the start of treatment-3290 $(76 \%)$ in the non-EPACS group and $616(86 \%)$ in the EPACS group. Work-up was ordered before the day of the first visit in 510 of $3290(16 \%)$ non-EPACS patients and 207 of $616(34 \%)$ EPACS patients $(p<0.001)$. Until the start of treatment, patients in the non-EPACS group made a median of 2 (IQR 1-3) and mean of 2.2 (standard deviation 1.4) visits to the hospital, while patients in the EPACS group made a median of 2 (IQR 1-3) and a mean of 2.0 (standard deviation 1.1$)$ visits $(p=0.426)$.

\section{Primary Outcomes: Cancellation Rate, Time} to Treatment, and Conversion Rate

In the non-EPACS group, 504 of 4342 (12\%) patients cancelled their first visit, compared with 33 of $720(4.6 \%)$ in the EPACS group $(p<0.001)$ [Fig. 3a]. The cancellation rate was significantly lower in the EPACS group in the 
TABLE 1. Patient characteristics

\begin{tabular}{|c|c|c|c|}
\hline & non-EPACS $[n=4342]$ & EPACS $[n=720]$ & $p$-value \\
\hline Age, years & $59 \pm 15$ & $59 \pm 15$ & 0.883 \\
\hline Female sex & 2905 (67) & $459(62)$ & 0.009 \\
\hline Type of new visit & & & 0.004 \\
\hline In-person & $3511(81)$ & $565(77)$ & \\
\hline Telehealth & $814(19)$ & $172(23)$ & \\
\hline Surgical service & & & $<0.001$ \\
\hline Colorectal & $875(20)$ & $211(29)$ & \\
\hline Gastric and mixed tumor & $973(23)$ & $277(38)$ & \\
\hline Gynecology & $1360(31)$ & $180(24)$ & \\
\hline Hepatopancreatobiliary & $1117(26)$ & $69(9.4)$ & \\
\hline Tumor site at diagnosis & & & $<0.001$ \\
\hline Pancreas & $607(14)$ & $50(6.8)$ & \\
\hline Colon & $460(11)$ & $90(12)$ & \\
\hline Ovarian & $381(8.8)$ & $53(7.2)$ & \\
\hline Rectal & $204(4.7)$ & $63(8.5)$ & \\
\hline Sarcoma & $242(5.6)$ & $121(6.4)$ & \\
\hline Endometrial & $211(4.9)$ & $35(4.7)$ & \\
\hline Stomach & $109(2.5)$ & $39(5.3)$ & \\
\hline Melanoma & $279(6.5)$ & $0(0)$ & \\
\hline Uterus & $131(3.0)$ & $17(2.3)$ & \\
\hline Liver & $186(4.3)$ & $12(1.6)$ & \\
\hline Cervical & $111(2.6)$ & $15(2.0)$ & \\
\hline Appendiceal & $68(1.6)$ & $19(2.6)$ & \\
\hline Other & $1336(31)$ & $223(30)$ & \\
\hline
\end{tabular}

Categorical data are expressed as $n(\%)$ and continuous data are expressed as mean
Colorectal and Gastric and Mixed Tumor Services $(p<0.001$ for each) and non-significantly lower in the Gynecology and Hepatopancreatobiliary Services. Among patients who had started treatment by the time of data analysis, the median time from first visit to the start of treatment was 19 days (IQR 11-30) in the non-EPACS group and 17 days (IQR 10-29) in the EPACS group ( $p=0.086$ ) [Fig. 3b]. Although not significantly different, the median time to treatment was shorter in the EPACS group in the Colorectal, Gynecology, and Hepatopancreatobiliary Services, but not in the Gastric and Mixed Tumor Service. Among patients who completed their first visit $\geq 3$ months before data collection, the conversion rate was 1489 of $2955(50 \%)$ in the non-EPACS group and 290 of $524(55 \%)$ in the EPACS group ( $p=0.037$ ) (Fig. 3c). The conversion rate was significantly higher in the EPACS group in the Hepatopancreatobiliary Service $(p=0.001)$ and non-significantly higher in the EPACS groups in the other services.

\section{Secondary Outcomes: Care Team and Patient Surveys}

The response rate for the electronic survey among APPs, OCs, and surgeons was 17 of $17(100 \%), 17$ of $21(81 \%)$, and 16 of $23(70 \%)$, respectively. EPACS increased the workload for 3 of 17 (18\%) APPs, but decreased it for 28 of $50(56 \%)$ responding care providers, and stayed the same for the remaining (Fig. 4a). EPACS improved patient interaction for 41 of 50 responding care providers; this proportion was lowest among OCs, at 10 of $17(59 \%)$. Because of EPACS, outpatient clinic was perceived as more efficient by 27 of 33 (82\%) APPs and surgeons. Of 58 phone calls for the patient survey, 31 patients answered, while 29 (94\%) responded that EPACS helped reduce anxiety (26 of 29 by 8 points or more) and helped them to feel better prepared for the first visit ( 27 of 29 by 8 points or more) [Fig. 4b].

\section{DISCUSSION}

This quality improvement study showed that the implementation of a clinical streamlining process for new patients, namely EPACS, improved health care at our 
(a)

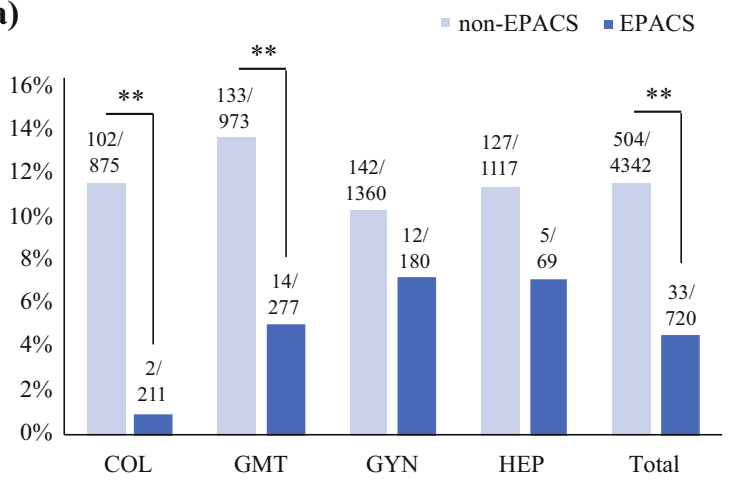

(c)

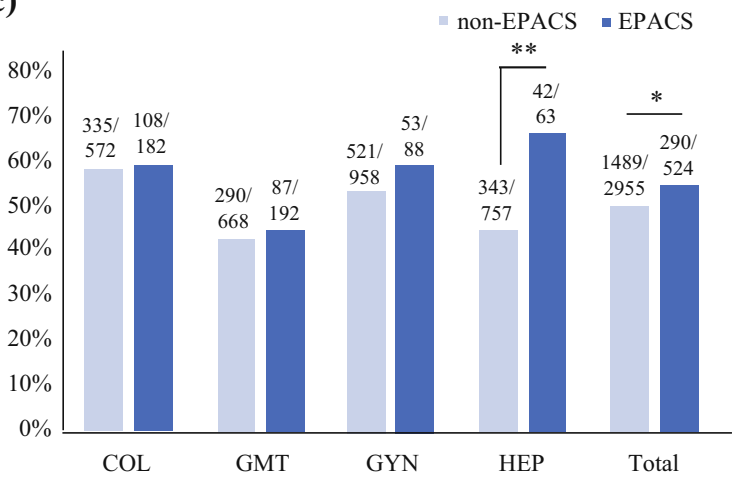

(b)

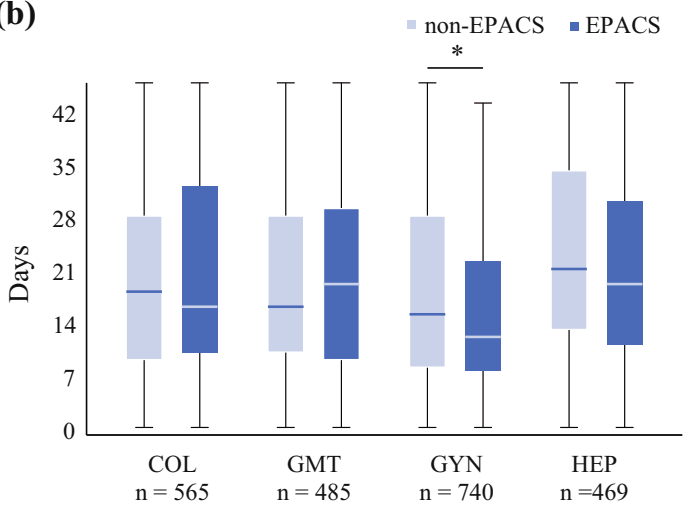

months of follow-up. EPACS Enhanced Patient Clinical Streamlining,

FIG. 3. Comparison of quality indicators between patients who received an EPACS call and those who did not. (a) Cancellation rate; (b) time to treatment among patients who had started treatment by the time of data analysis; (c) conversion rate among patients with $\geq 3$ $C O L$ colorectal, GMT gastric and mixed tumor, GYN gynecology, HEP hepatopancreatobiliary

cancer center. The EPACS pre-visit call was associated with better patient retention, as shown by a two-thirds reduction in cancellations of the first visit, and a $10 \%$ increase in the number of patients who stayed at our institution for treatment. Most importantly, timeliness of care was improved, measured as a 2-day reduction in time from first visit to treatment. EPACS also improved the care experience for both patients and providers according to our survey responses. EPACS improves healthcare outcomes by reorganizing care using existing resources and enabling early engagement with the patient by a member of their care team.

EPACS was adopted immediately after launch, as the percentage of new patients receiving an EPACS call did not increase. Acceleration in EPACS usage was not actively encouraged and the rate of EPACS calls (14\%) likely reflects barriers to adoption. EPACS requires some experience of the APP with the cancer of interest and the treating surgeon. Furthermore, although $82 \%$ (14/17) of APPs did not feel that the workload increased, EPACS requires a change in work processes and takes time; approximately half of the APPs did not have administrative time scheduled. As the median wait time for the first visit was 5 calendar days (IQR 3-7), it was sometimes

challenging for the APP to find time in their schedule for the call within that period. Interestingly, $34 \%$ of patients received the EPACS call only 1 day before the first visit. Despite these barriers, the APPs implemented the new work process into their routine, perhaps motivated by potential positive effects for patients, surgeons, and themselves. The coronavirus disease 2019 (COVID-19) pandemic in New York City, and related hospital policy and workflow changes, happened after EPACS was launched. Although the pandemic caused an increase in cancellations and time from first visit to treatment, the effect of EPACS on these outcomes was similar before and during the pandemic.

A compelling difference in outcomes was the two-thirds reduction in cancellation rates in the EPACS group (12\% vs. $4.2 \%, p<0.001)$. At other institutions, appointment reminders by staff have been introduced to reduce noshows from 23 to $14 \% .^{8}$ At our cancer center, the number of no-shows is negligible, but patients mostly cancel their appointments because they selected a competitor institution. One of the surveyed patients said that "the call was instrumental in changing from another hospital to yours." Given that there are several thousand first visits per year at our surgery department, if EPACS were to be deployed for 
FIG. 4. Survey responses regarding the care experience. (a) Care providers; (b) patients. Survey respondents ranked answers from 0 , indicating that EPACS did not improve the indicated experience at all, to 10 , meaning that it helped very much. EPACS Enhanced Patient Clinical Streamlining, $A P P$ advanced practice provider, $O C$ office coordinator, $M D$ medical doctor (surgeon)

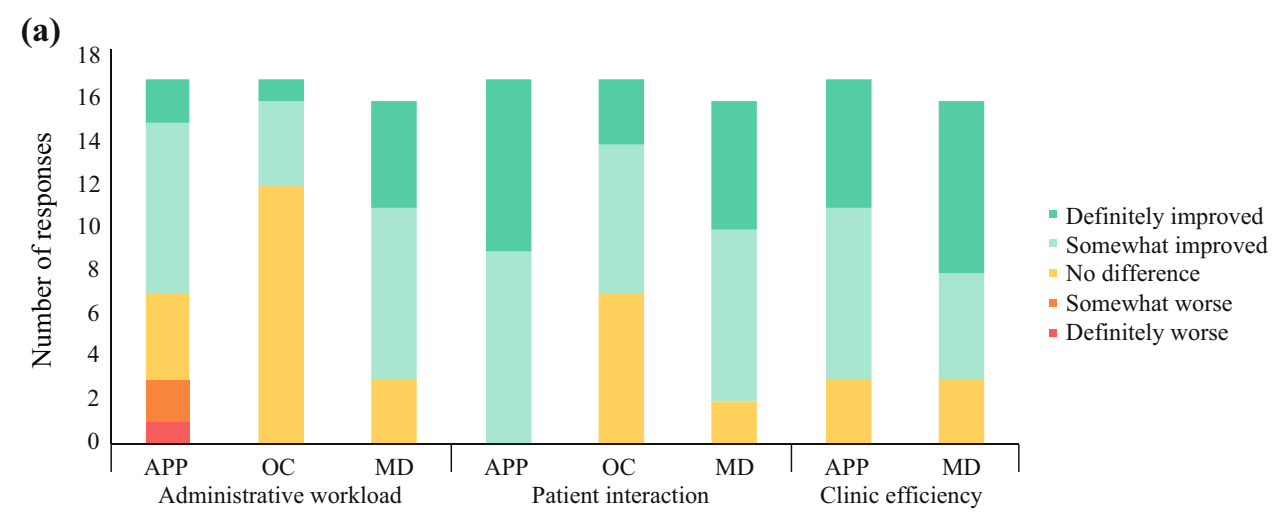

(b)

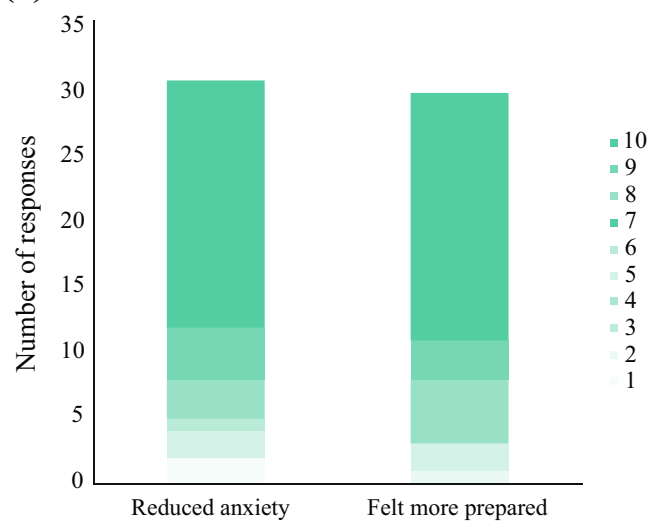

all, a 7-8\% reduction in cancellations could result in an additional several hundred initial consultations. If EPACS for all new patients is not achievable, patients at higher risk for cancellation could be targeted. Similarly, the number of patients who received treatment increased by $10 \%$ in the EPACS group, which could result in treating an additional few hundred patients a year at our institution. For these reasons, we concluded that EPACS increased the effectiveness of care, a core dimension of health care quality. ${ }^{9}$

EPACS also improved with regard to another subdimension of health care quality, timeliness, ${ }^{9}$ as indicated by the 2-day reduction in time to treatment, although this was not significant $(p=0.086)$. Time to treatment was not shorter in the EPACS group of the Gastric and Mixed Tumor Service, which treats sarcoma, melanoma, and gastric cancer, among other tumor types. The melanoma care team has no APPs, therefore all new patients with melanoma were included in the non-EPACS group, representing $30 \%$ of non-EPACS patients in this service. In addition, melanoma patients can often be treated in an outpatient setting with a shorter time to treatment, reducing the time to treatment in the non-EPACS group. Broader adoption of EPACS is likely to be associated with a reduction in time to treatment as a result of both earlier work-up orders and earlier collection of documents, radiological imaging, pathological slides, etc. related to prior diagnosis. If EPACS for all new patients in not achievable, patients at highest risk of prolonged time to treatment could be targeted, such as older patients, Black patients, or those with multiple comorbidities or stage I disease. $^{4}$

The EPACS call-associated reduction in pre-visit anxiety in $94 \%$ (29/31) of patients is an important outcome, as $59 \%$ of cancer patients were highly anxious before the initial consultation. ${ }^{3}$ The fact that the EPACS call helped the patient feel more prepared may have contributed to the improved patient interaction noted by APPs and surgeons. Some unsolicited feedback received during the patient survey included: "I was terrified and the APP calmed me"; "that's the first night I got a good night's sleep actually"; "I felt there was a plan in place"; and "everyone should have this". If resources for EPACS are not available, a web-based tailored educational intervention might be an alternative, as such tools have also been shown to reduce anxiety and increase patient satisfaction. ${ }^{10}$ Physician satisfaction also improved, as EPACS was considered to reduce workload and improve outpatient clinic efficiency. As organizational approaches to streamline clinical work processes have been found to reduce burnout scores, ${ }^{6}$ EPACS may help prevent physician burnout. 
Most importantly, the implementation of this new workflow was appreciated by our patients. At a highly anxious moment, a new cancer diagnosis, this approach provided comfort, information, and reassurance that help was on the way and was being prioritized for that individual. Furthermore, APPs and surgeons noted a smoother, more efficient, and less cumbersome cancer work-up that saved time and frustration and allowed them to focus more on patient care. Generalizability of EPACS to other care centers might be limited because every department and institution has their own workflow. However, the underlying concept of a pre-initial consultation connection and engagement with the patient is generalizable. The outcome measures of cancellation and conversion rate might be less relevant for health care systems in less competitive environments than New York City; however, EPACS might be similarly effective in reducing no-show rates. ${ }^{8}$

A limitation of the current study was lack of randomization and the lack of quotas for the number of phone calls per APP. APPs with a positive attitude towards EPACS may have been more likely to make more EPACS calls, and the APP's attitude could have contributed to the effects seen. If the latter is true, the APPs should receive even more credit for the improvements associated with EPACS. The participating APPs' knowledge about the patient's disease and the ability to ease patients' anxiety during EPACS calls was a strength of this intervention, partly resulting from our institution's high volume of cancer patients. EPACS is currently being expanded throughout our institution.

\section{CONCLUSIONS}

The implementation of EPACS, a reorganization of care using existing resources that enables an early connection prior to a first visit with an experienced provider, improved the effectiveness, timeliness, and physician and patient satisfaction with health care at our cancer center.

Supplementary Information The online version contains supplementary material available at https://doi.org/10.1245/s10434021-11126-3.

ACKNOWLEDGMENT The authors thank the patients, APPs, OCs, and surgeons who participated in this study, and Jessica Moore, MS, for editing. This study was supported in part by an NCI Cancer Center Support Grant (P30 CA008748) to the institution for core resources.
DISCLOSURES Nadeem Abu-Rustum has received research grants from Stryker/Novadaq and GRAIL, paid to the institution and separate from the submitted work. Julio Garcia Aguilar has received honoraria from Intuitive Surgical, Johnson and Johnson, and Medtronic, and owns stock in Intuitive Surgical. Jeffrey Drebin owns stock in Alnylam, Ionis, and Arrowhead, and his spouse is employed by American Regent Pharmaceuticals. Elvira L. Vos, Jessica S. Cho, Joseph Schmeltz, Nick Teri, Ethel B. Law, Kathleen Paisley, Aaron Begue, Helen Loumeau, Sherri H. Suozzo, Latasha Anderson-Dunkley, Ginger J. Gardner, Elizabeth Jewell, Samuel Singer, William R. Jarnagin, and Vivian E. Strong have no financial interests to disclose.

\section{REFERENCES}

1. Pfister DG, Rubin DM, Elkin EB, et al. Risk adjusting survival outcomes in hospitals that treat patients with cancer without information on cancer stage. JAMA Oncol. 2015;1(9):1303-10.

2. Resio BJ, Chiu AS, Hoag JR, et al. Motivators, barriers, and facilitators to traveling to the safest hospitals in the united states for complex cancer surgery. JAMA Netw Open. 2018;1(7):e184595.

3. Bronner MB, Nguyen MH, Smets EMA, van de Ven AWH, van Weert JCM. Anxiety during cancer diagnosis: examining the influence of monitoring coping style and treatment plan. Psychooncology. 2018;27(2):661-7.

4. Bilimoria KY, Ko CY, Tomlinson JS, et al. Wait times for cancer surgery in the United States: trends and predictors of delays. Ann Surg. 2011;253(4):779-85.

5. Neal RD, Tharmanathan P, France B, et al. Is increased time to diagnosis and treatment in symptomatic cancer associated with poorer outcomes? Systematic review. $B r \quad J$ Cancer. 2015;112(Suppl 1):S92-107.

6. West CP, Dyrbye LN, Erwin PJ, Shanafelt TD. Interventions to prevent and reduce physician burnout: a systematic review and meta-analysis. Lancet. 2016;388(10057):2272-81.

7. Ogrinc G, Davies L, Goodman D, Batalden P, Davidoff F, Stevens D. SQUIRE 2.0-standards for quality improvement reporting excellence-revised publication guidelines from a detailed consensus process. J Am Coll Surg. 2016;222(3):317-23.

8. Parikh A, Gupta K, Wilson AC, Fields K, Cosgrove NM, Kostis JB. The effectiveness of outpatient appointment reminder systems in reducing no-show rates. Am J Med. 2010;123(6):542-8.

9. Busse RKN, Panteli D, et al editors. Improving healthcare quality in Europe: characteristics, effectiveness and implementation of different strategies. Copenhagen: European Observatory on Health Systems and Policies; 2019.

10. Nguyen MH, Smets EM, Bol N, et al. Tailored web-based information for younger and older patients with cancer: randomized controlled trial of a preparatory educational intervention on patient outcomes. J Med Internet Res. 2019;21(10):e14407.

Publisher's Note Springer Nature remains neutral with regard to jurisdictional claims in published maps and institutional affiliations. 